\title{
CARACTERÍZACIÓN PETROGRAFÍCA Y QUÍMICA DE ROCAS DE CORTEZA OCEÁNICA DEL COMPLEJO QUEBRADAGRANDE Y COMPARACIÓN CON ROCAS DE LA UNIDAD DIABASAS DE SAN JOSÉ DE URAMA
}

Gabriel Rodríguez G. ${ }^{1}$; Lina María Cetina T. ${ }^{1}$

DOI: http://dx.doi.org/10.18273/revbol.v38n3-2016001 (ㄷ) (1) Ð

Forma de citar: Rodríguez, G., y Cetina, L.M. 2016. Caracterización petrográfica y química de rocas de corteza oceánica del Complejo Quebradagrande y comparación con rocas de unidad Diabasas de San José de Urama. Boletín de Geología, 38(3): 15-29.

\section{RESUMEN}

El Complejo Quebradagrande está conformado por "bloques litológicos" de rocas sedimentarias de origen marino, vulcanitas de arco (tobas, andesitas, basaltos) y bloques de corteza oceánica (diabasas y basaltos). Se encuentra limitado al E por la Falla San Jerónimo que lo separa del Complejo Cajamarca y al W por la Falla Silvia - Pijao, que lo separa del Complejo Arquía. Los "bloques" de corteza oceánica están representados por diabasas, basaltos variolíticos y basaltos y diabasas deformadas, con texturas intergranular, ofítica-subofítica y variolítica, constituidos principalmente por plagioclasa y clinopiroxeno y como accesorios titanita y opacos. Las diabasas y basaltos del Complejo Quebradagrande son rocas básicas con valores de $\mathrm{SiO}_{2}$ entre 44,95\% y $50,80 \%$, presentan concentraciones altas de $\mathrm{MgO}$ en el rango entre 5,42 y 8,9\%, el $\mathrm{Fe}_{2} \mathrm{O}_{3}$ varía entre 7,98 y $14,83 \%$, los contenidos de $\mathrm{CaO}$ son altos entre 7,55\% y 13,2\%, los valores de $\mathrm{TiO}_{2}$ en la mayoría de las muestras están entre 1 y $2 \%$, tienen una baja relación del álcalis vs $\mathrm{SiO}_{2}(0,03$ a $0,10 \mathrm{wt} \%)$ y los valores de $\mathrm{K}_{2} \mathrm{O}$ son bajos de $0,07 \%$ a $0,47 \%$. Corresponden a la serie toleítica rica en $\mathrm{Mg}$, poco diferenciados y de afinidad subalcalina y tienen relaciones $\mathrm{Zr} / \mathrm{Nb}$ mayores a $30,(\mathrm{La} / \mathrm{Sm}) \mathrm{N}$ menor a 1 y patrones de tierras raras que sugieren afinidad con basaltos tipo N-MORB, excepto dos muestras (VR-364R y VR-384R) que exhiben patrones similares a los E-MORB. Se diferencian de las Diabasas de San José de Urama en que estas últimas presentan valores $\mathrm{Zr} / \mathrm{Nb}$ entre 10 y 15, y la relación $(\mathrm{La} / \mathrm{Sm}) \mathrm{N}$ tienen valores menores y mayores a 1 , con patrones de tierras raras planos similares a basaltos de tipo T-MORB. Estos resultados sugieren que corresponden a cortezas oceánicas distintas, no coetáneas, con rocas de arco asociadas que presentan diferencias en la composición química, que no son correlacionables, exceptuando las muestras VR-364R y VR-384R, que exhiben similitud con la geoquímica de las Diabasas de San José de Urama.

Palabras claves: Colombia, Complejo Quebradagrande, unidad Diabasas de San José de Urama, texturas "diabásicas", geoquimica.

\section{PETROGRAPHIC AND CHEMICAL CHARACTERIZATION OF OCEANIC CRUST ROCKS FROM THE COMPLEJO QUEBRADAGRANDE AND COMPARISON WITH THE DIABASAS DE SAN JOSÉ DE URAMA UNIT}

\begin{abstract}
The Complejo Quebradagrande unit is formed by "lithological blocks" of sedimentary rocks of marine origin, arc vulcanites (tuffs, andesites and basalts) and oceanic crust "blocks" (basalts and diabases). It is limited to the East by the San Jeronimo fault that separates it from the Complejo Cajamarca unit and to the West by the Silvia-Pijao fault that separates it from the Complejo Arquia unit. The "blocks" of oceanic crust are represented by diabases, variolitic basalts and deformed diabases and basalts, with intergranular, ophitic-subophitic and variolitic textures, mainly composed of plagioclase and clinopyroxene and, as accessory minerals, titanite and opaques. The diabases and basalts of the Complejo Quebradagrande unit are basic rocks with $\mathrm{SiO}_{2}$ values between $44.95 \%$ and $50.80 \%$, high concentrations of $\mathrm{MgO}$ in the range between 8.9 and $5.42 \%, \mathrm{Fe}_{2} \mathrm{O}_{3}$ varies
\end{abstract}

${ }^{1}$ Servicio Geológico Colombiano, grodriguez@sgc.gov.co; linacetina0526@gmail.com 
between 14.83 and $7.98 \%, \mathrm{CaO}$ contents are high between $7.55 \%$ and $13.2 \%$, values of $\mathrm{TiO}_{2}$ in the majority of samples are between 1 and $2 \%$, low ratio of alkalis $\mathrm{vs}_{\mathrm{SiO}_{2}}(0.03$ to $0.10 \mathrm{wt} \%)$ and low $\mathrm{K}_{2} \mathrm{O}$ values between $0.07 \%$ and $0.47 \%$. The rocks correspond to the high in $\mathrm{Mg}$ tholeiitic series, little differentiated and of subalkaline affinity, and have greater than $30 \mathrm{Zr} / \mathrm{Nb}$ ratios, $(\mathrm{La} / \mathrm{Sm}) \mathrm{N}$ less than 1 and rare earths patterns that suggest affinity with N-MORB type basalts, except two samples (VR-364R and VR-384R) that exhibited patterns similar to the E-MORB. The rocks of the Complejo Quebradagrande unit differ from the Diabasas de San Jose de Urama unit in that the rocks of the latter have $\mathrm{Zr} / \mathrm{Nb}$ values between 10 and 15 , and that the ratios $(\mathrm{La} / \mathrm{Sm}) \mathrm{N}$ present values greater and lower than 1, with flat rare earths patterns similar to type T-MORB basalts. These results suggest that the rocks of these two units correspond to different, not contemporary oceanic crusts, with associated arc rocks that present a difference in the chemical composition, that are not correlatable, except for samples VR-364R and VR-384R, which exhibit a geochemical similarity with the Diabasas de San José de Urama unit.

Keywords: Colombia, Complejo Quebradagrande, Diabasas de San José de Urama unit, “diabasic” textures, geochemistry

\section{INTRODUCCIÓN}

La primera descripción geológica del Complejo Quebradagrande fue realizada por Grosse (1926) bajo el nombre de "formación Porfirítica", posteriormente Botero (1963) utilizó el término Formación Quebradagrande y estableció la sección tipo a lo largo de la quebrada Grande, reconociendo un conjunto sedimentario y otro de rocas verdes volcánicas; González (1976) dividió la Formación Quebradagrande en un miembro sedimentario y otro volcánico; Álvarez (1987) elevó el estatus de la unidad a Grupo; y González (2001) definió con el nombre de Complejo Quebradagrande a la unidad que agrupa rocas volcánicas y sedimentarias que afloran como una franja larga, estrecha y discontinua, en el flanco oeste de la Cordillera Central al occidente de la Falla San Jerónimo y al este de la Falla Silvia-Pijao.

El Complejo Quebradagrande está conformado por "bloques litológicos" de rocas sedimentarias de origen marino (lodolitas, arenitas arcósicas, cuarzoarenitas, chert y conglomerados de variada composición), vulcanitas de arco (tobas, andesitas, basaltos), y fragmentos de corteza oceánica (gabros, basaltos y diabasas) (Moreno et al., 2008; Villagómez et al., 2011; Rodríguez y Zapata, 2013).

Las diabasas del Complejo Quebradagrande son el objeto central del presente artículo; se describen las características petrográficas y químicas y se compara con la química de rocas de corteza oceánica de la unidad Diabasas de San José de Urama descrita por Rodríguez y Arango (2013a) para determinar si existe o no relación genética entre dichas rocas, siendo ésta la unidad de diabasas más cercana geográficamente, pero geológicamente separada por fallas regionales del Sistema Romeral, y que además, presenta relaciones espaciales similares con vulcanitas de arco (FIGURA 1). Las rocas de corteza oceánica se encuentran en menor cantidad que otras litologías dentro del Complejo Quebradagrande, siendo poco documentadas y estudiadas en trabajos anteriores.

El trabajo demuestra la existencia, dentro del Complejo Quebradagrande, de rocas diabásicas de corteza oceánica, con características químicas que sugieren para la mayoría de ellas un ambiente geotectónico diferente al de las Diabasas de San José de Urama y compara, en algunos gráficos, los campos de rocas de arco asociadas a ambas cortezas.

\section{MARCO GEOLÓGICO}

El Complejo Quebradagrande se encuentra en el flanco occidental de la Cordillera Central colombiana, limitado al E por la Falla San Jerónimo que lo separa del Complejo Cajamarca, el cual incluye pizarras, esquistos verdes, esquistos negros, cuarcitas, mármoles, anfibolitas y migmatitas que registran un evento metamórfico Triásico; y al W por la Falla Silvia-Pijao que lo separa del Complejo Arquía, unidad que comprende esquistos anfibólicos y sericíticos, esquistos verdes y negros, cuarcitas y neises, junto a anfibolitas y metagabros con edades Ar-Ar principalmente triásicas y K/Ar cretácicas (Rodríguez y Arango, 2013b). Se ubica regionalmente en la zona de colisión entre el basamento metamórfico de la Cordillera Central, representado por bloques metamórficos de variadas edades (Cretácico, Jurásico Tardío, Triásico, Pérmico y Paleozoico) y el basamento oceánico de la Cordillera Occidental, representado por basaltos de corteza oceánica y rocas volcánicas de arco que en el norte de la Cordillera Occidental se han denominado Formación Barroso y Diabasas de San José de Urama (Rodríguez y Arango, 2013a) (FIGURA 1). 


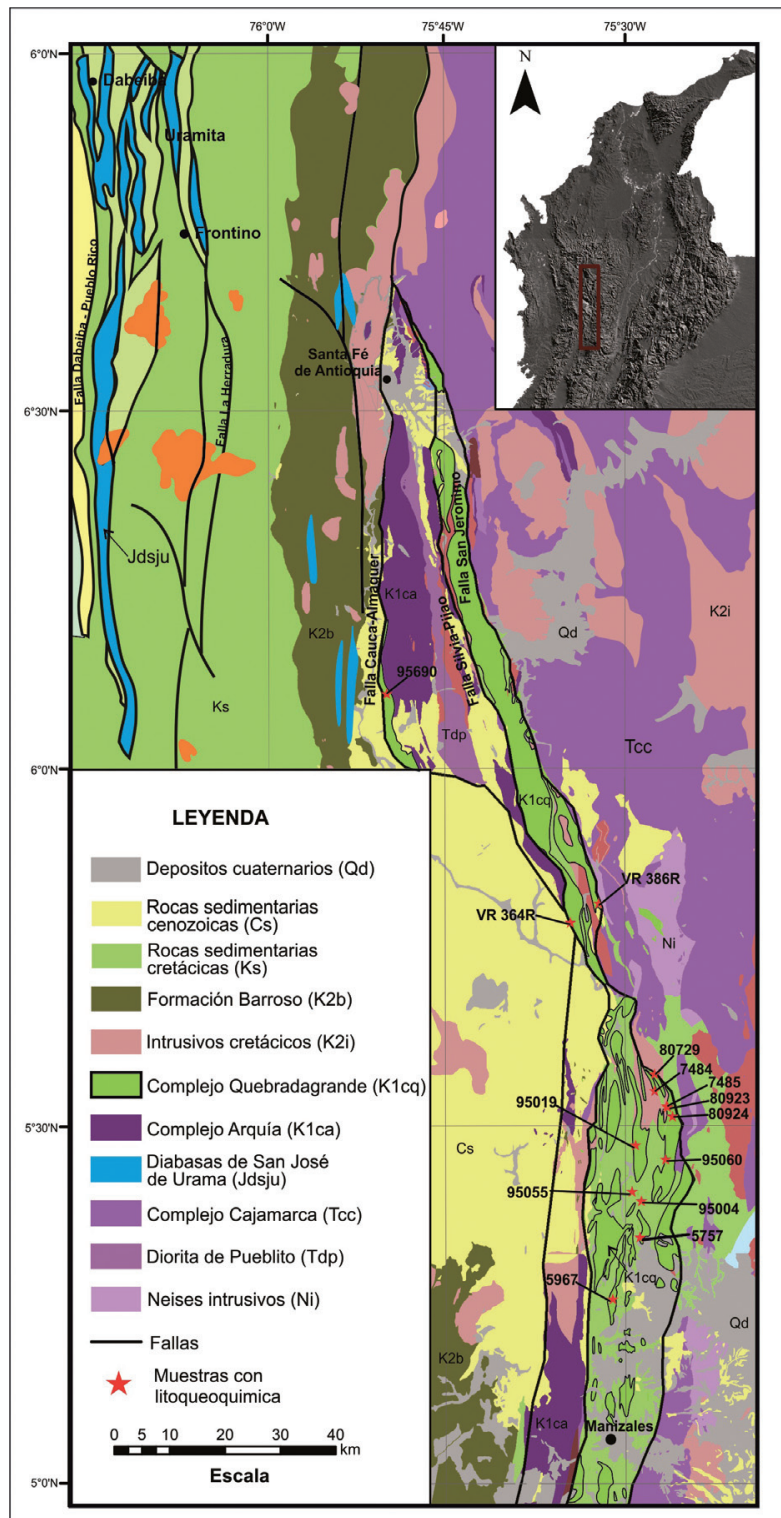

FIGURA 1. Localización regional del Complejo Quebradagrande y ubicación de muestras de rocas diabásicas con petrografía y análisis químicos.

La unidad Diabasas de San José de Urama fue propuesta por Mejía y Salazar (1987) y redefinida por Rodríguez y Arango (2013a), quienes la limitan a los bloques tectónicos de diabasas y basaltos almohadillados (pillow lavas), con texturas ofíticas, subofíticas, intergranulares, variolíticas e intersectales que afloran en el segmento norte de la Cordillera Occidental, entre las fallas Cauca-Almaguer y Dabeiba-Pueblo Rico. Químicamente corresponden a toleítas bajas en $\mathrm{K}$, con un patrón de elementos traza y REE asociada a una fuente mantélica de tipo T-MORB, con una edad de 155,1 111,2 Ma obtenida por el método Ar-Ar en roca total, que la ubica en el Jurásico SuperiorCretácico Inferior (Rodríguez y Arango, 2013a).
La Formación Barroso fue propuesta por Álvarez y González (1978), quienes en la definición original agruparon todas las vulcanitas que afloran a lo largo del borde oriental de la Cordillera Occidental en Antioquia, incluyendo las rocas que conforman las Diabasas de San José de Urama. Rodríguez y Arango (2013a) redefinen la Formación Barroso y la limitan únicamente a las rocas volcano-sedimentarias como basaltos, andesitas con texturas porfídicas y amigdalares, aglomerados y tobas de series toleíticas y calco alcalinas medias en K, generadas en ambiente de arco y con edades entre 88 y $115 \mathrm{Ma}$ (Turoniano-Apiano).

El Complejo Quebradagrande ha sido objeto de múltiples interpretaciones entre las que se destacan las presentadas por Nivia et al., (1996) y Álvarez (1987), donde el Complejo (en su parte occidental) representaría un arco volcánico. Posteriormente Nivia et al., (2006) plantean un modelo petrogenético que podría representar una posible cuenca intracratónica marginal del Cretáceo Temprano, generándose las rocas en un ambiente de suprasubducción. Villagómez et al., (2011) consideran que el Complejo Quebradagrande tiene rocas afines a MORB y rocas relacionadas a arco, indicando que el arco se desarrolló a través de una corteza oceánica o una corteza transicional atenuada que bordeaba el margen continental. Para Rodríguez y Zapata (2013) el Complejo Quebradagrande representa parte del arco Barroso-Sabanalarga segmentado por el sistema de fallas de Cauca-Romeral. Recientemente se ha postulado un modelo de un arco de islas exótico el cual colisiona con el continente (Jaramillo et al., 2014).

$\mathrm{Al}$ interior del Complejo Quebradagrande se reconocen litodemas sedimentarios con aporte de material volcánico proveniente del arco que muestra una población de circones con edades del Cenomaniano-Aptiano (95-115 Ma) y aporte de dos poblaciones de circones detríticos provenientes de la Cordillera Central, Triásico-Pérmico tardío (220-270 Ma) y Ordovícico-Cámbrico (460$500 \mathrm{Ma}$ ) (Pardo et al., 2011). Recientemente Jaramillo et al. (2015) presentan edades $\mathrm{U} / \mathrm{Pb}$ para rocas de arco del Complejo Quebradagrande entre el Albiano y el Campaniano, sugiriendo varios pulsos magmáticos de arco. El registro fósil predominante es de edad Albiano, y se tiene una datación $\mathrm{U} / \mathrm{Pb}$ en una toba que se considera afín con las rocas de arco del Complejo Quebradagrande, la cual arrojó una edad de 114,3 $\pm 3,8$ Ma (Villagómez et al., 2011), además una edad U/Pb de 112,9 Ma publicada por Cochrane et al. (2014) en un dique de diorita. En este contexto, la edad sugerida para las vulcanitas de arco del Complejo Quebradagrande está en el rango Aptiano-Albiano y para las diabasas de corteza oceánica no se conoce la edad. 


\section{MATERIAL Y METODOS}

Se realizó una revisión petrográfica de 195 secciones delgadas colectadas por INGEOMINAS (actual Servicio Geológico Colombiano- SGC) en los trabajos regionales de cartografía geológica (planchas 130, 146, 166, 206 y el proyecto Cauca Romeral Zona Norte). Se separaron las rocas con texturas ofíticas, subofíticas, intergranulares, intersectales y variolíticas, siguiendo las consideraciones de Rodríguez y Arango (2013a), quienes asocian dichas texturas a rocas de corteza oceánica, y se determinó la distribución espacial de las mismas dentro del Complejo Quebradagrande. Se separaron las tobas y rocas andesíticas y basálticas con texturas porfídicas que muestran características químicas asociadas a un ambiente de arco, las cuales no son el objeto principal de este trabajo.

Se realizó análisis químico a 12 muestras de diabasas en el laboratorio químico del SGC, sede Bogotá; las muestras fueron seleccionadas considerando el menor grado de alteración y deformación dentro de una unidad que está deformada y alterada debido a su localización estructural entre las fallas San Jerónimo al este y Silvia Pijao al oeste; además, se tomaron dos análisis de muestras de diabasas del proyecto Cauca-Romeral (GEOESTUDIOS-INGEOMINAS, 2005) elaborados en el laboratorios ACME (Canadá) y cuya metodología y límites de detección se describen en dicho informe, para un total de 14 análisis seleccionados en roca total. Para determinar los contenidos en óxidos mayores en el laboratorio químico del SGC sede Bogotá, se utilizó el método de fluorescencia de rayos $\mathrm{X}$ en base seca, incluyendo los elementos traza: V, Mo, Nb, Ta, W, Zr y Hf, y para el resto de elementos traza y tierras raras se utilizó el equipo de espectrometría de masas con plasma acoplado inductivamente (ICP-MS) cuyos límites de detección se pueden consultar en la página Web del SGC. Para la interpretación de los óxidos mayores se hizo el recalculo, teniendo en cuenta los valores de LOI (perdidas por ignición).

\section{CARACTERISTICAS PETROGRÁFICAS DE LAS DIABASAS DEL COMPLEJO QUEBRADAGRANDE}

Las 14 muestras con texturas "diabásicas" corresponden a diabasas, basaltos variolíticos y milonitas y cataclásitas según basaltos y diabasas (TABLA 1). La relación de fábrica entre la plagioclasa y el clinopiroxeno evidencian las texturas "diabásicas", las cuales son comparables a la composición y textura descritas en unidades de la Cordillera Occidental como Diabasas de San José de Urama (Rodríguez y Arango, 2013a), Formación Volcánica (Aspen et al., 1984, Rodríguez et al., 2010) y Grupo Diabásico (Nelson, 1957). Las rocas se caracterizan por presentar un entrecruzamiento de plagioclasas tabulares, dejando espacios intergranulares ocupados por cristales de clinopiroxeno (textura intergranular), clinopiroxeno poiquilítico con inclusiones de plagioclasa (textura ofitica-subofitica) y disposición en "ramillete" de clinopiroxenos con espacios que son ocupados por plagioclasa, en su mayoría alterada a agregados de saussurita (textura variolítica) (FIGURA 2).

TABLA 1. Composición modal y texturas de las diabasas del Complejo Quebradagrande.

\begin{tabular}{|c|c|c|c|c|c|c|c|c|c|c|c|c|c|}
\hline Muestra & PI & Cpx & Anf & Chl & Op & $\mathbf{Z r}$ & Ttn & Ep & Cal & Matriz & Ser & Textura & $\begin{array}{l}\text { Clasificación } \\
\text { Petrográfica }\end{array}$ \\
\hline 5757 & 63 & 35 & & & & & & & 2 & & $\mathrm{x}$ & Intergranular subofítica & Diabasa \\
\hline 5967 & 62 & 38 & & $\mathrm{x}$ & & & & $\mathrm{x}$ & & & $\mathrm{x}$ & Variolítica-intergranular & Basalto variolítico \\
\hline 7484 & 65 & 32 & & & 3 & & & & & $\mathrm{x}$ & & Ofítica y subofítica & Diabasa cataclástica \\
\hline 7485 & 65 & 2 & 30 & & 3 & & & & & & $\mathrm{x}$ & Alotriomórfica & Diabasa cataclástica \\
\hline 80729 & 42 & 35 & & $\operatorname{Tr}$ & $\operatorname{Tr}$ & $\operatorname{Tr}$ & $\operatorname{Tr}$ & & & 23 & & Alotriomórfica y ofítica & Diabasa con deformación \\
\hline 80923 & 59 & 15 & 10 & 10 & 3 & & $\mathrm{x}$ & 3 & & & & Afanítica intergranular & Diabasa espilitizada \\
\hline 80924 & 54 & 36 & & 5 & 3 & & $\mathrm{x}$ & 1 & 1 & & & Subofítica & Diabasa espilitizada \\
\hline 95004 & 19 & 5 & & 60 & 5 & & $\operatorname{Tr}$ & 1 & 5 & & & Afanítica & Filonita según Basalto \\
\hline 95019 & 61 & & 35 & & 3 & & 1 & 1 & & & & Variolítica & Basalto variolítico \\
\hline 95055 & 54 & 35 & 3 & 2 & 1 & & 2 & 3 & & & $\mathrm{x}$ & Alotriomórfica & Diabasa cataclástica \\
\hline 95060 & 52 & & 36 & 3 & 1 & & $\operatorname{Tr}$ & 5 & & & 3 & Porfirítica, matriz variolítica & Basalto variolítico \\
\hline 95690 & 51 & 47 & & 0 & & & 2 & & & & & Ofítica y subofítica & Diabasa con deformación \\
\hline VR386R & 53 & 42 & & & 4 & & & & & & & Variolítica & Diabasa milonitizada \\
\hline VR364R & 64 & 32 & & & 4 & & $\operatorname{Tr}$ & & & & & Porfirítica intergranular & Diabasa \\
\hline
\end{tabular}


A.

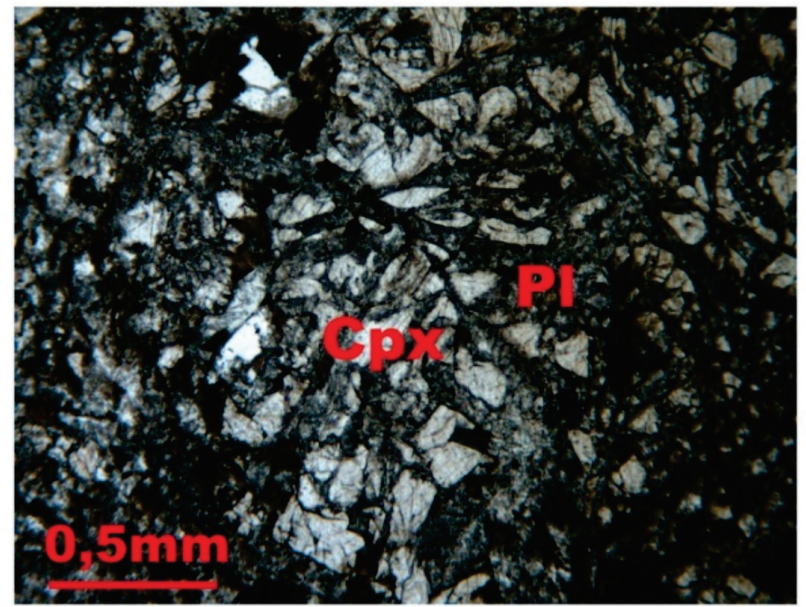

C.

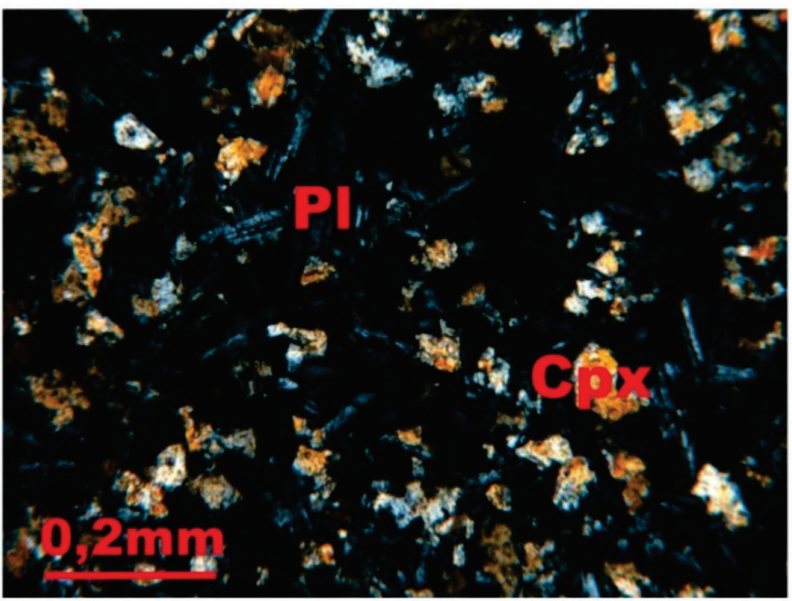

E.

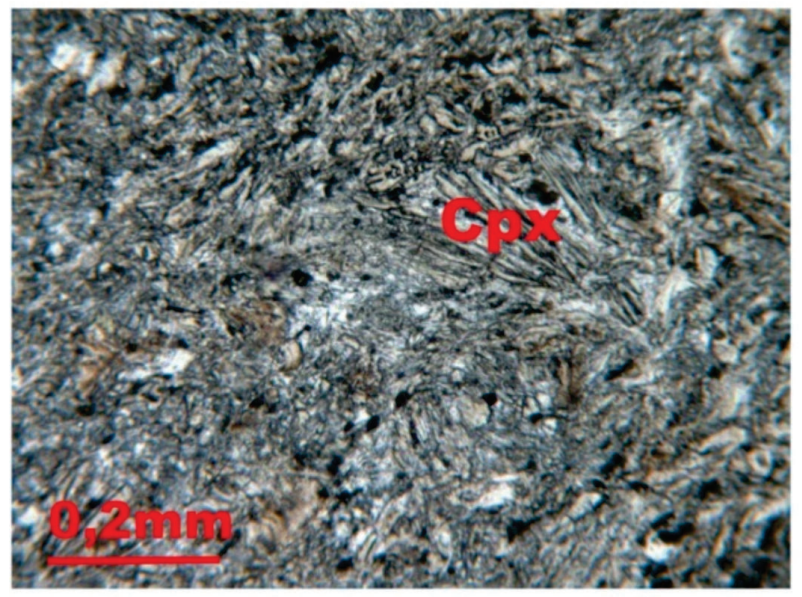

B.

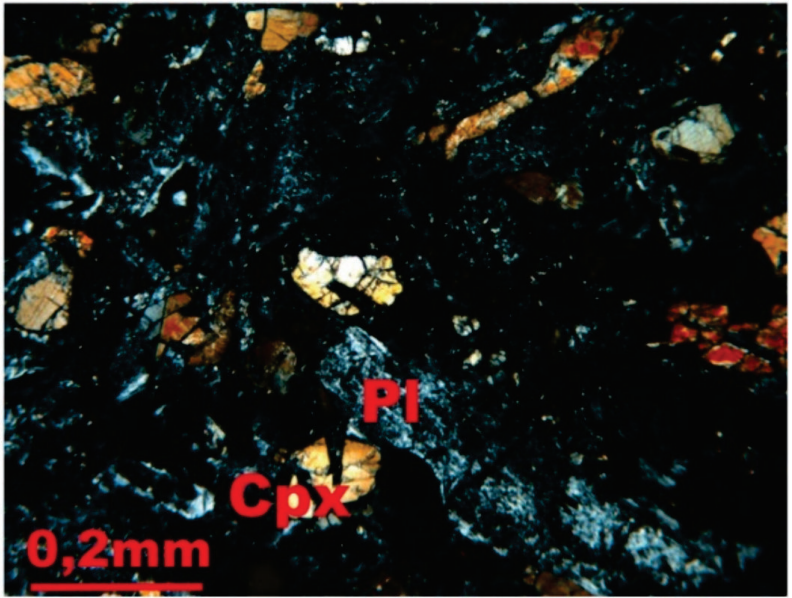

D.

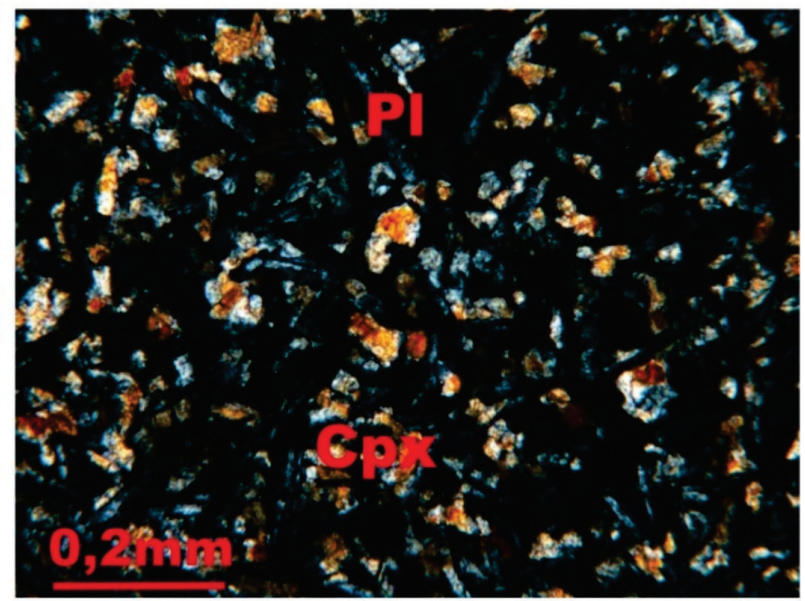

F.

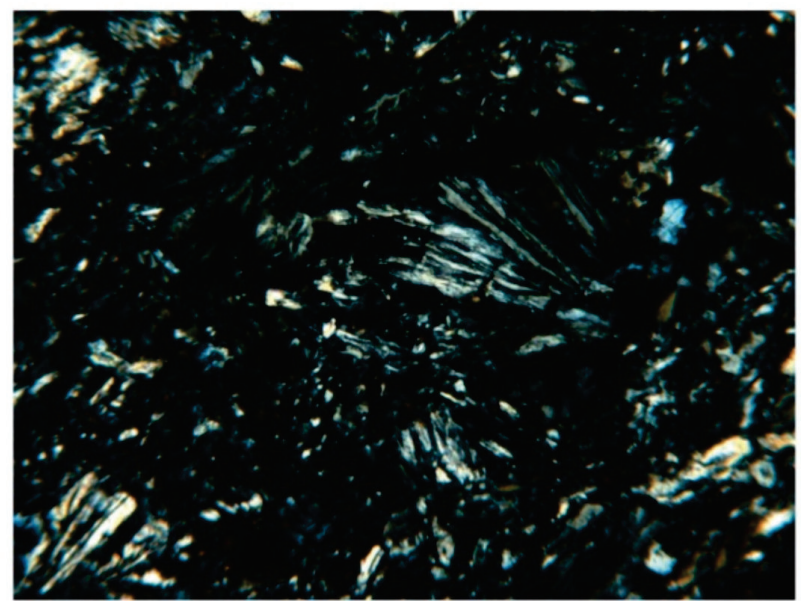

FIGURA 2. Características composicionales y texturales de diabasas del Complejo Quebradagrande: A. IGM 7484, plane polarized light (PPL), textura ofítica; B. IGM 5757, cross polarized light (XPL), textura intergranular; C. VR 364R; XPL, textura intergranular; D. VR 386R, XPL, textura intergranular; E y F. IGM 95060, PPL (Izq), XPL (Der), textura variolítica. 
Algunas diabasas tiene deformación dinámica frágil sobreimpuesta que destruye parcialmente la textura original de la roca, generando localmente texturas cataclásticas y micro-fallas con molido frágil de minerales, además presentan vetillas y micro-fracturas rellenas de epidota, cuarzo y calcita subordinada.

Los minerales constituyentes de las rocas son plagioclasa (en su mayoría con alteración saussurítica y en menor proporción a sericita) y clinopiroxeno (en algunas muestras uralitizado); los minerales accesorios corresponden a opacos y titanita alterada a leucoxeno; y como minerales de introducción se encuentran cuarzo, epidota, clorita y mica blanca, algunos rellenando fracturas y vetillas y otros rellenando amígdalas.

La plagioclasa varía entre 50 y $65 \%$; se encuentra generalmente alterada a agregados de saussurita $y$ en menor proporción a sericita, alteraciones que enmascaran las propiedades ópticas del mineral. Los cristales son euhedrales a subhedrales-tabulares y en agregados finos, incoloros a marrón terroso (por la alteración), de tamaño entre $0,3-0,1 \mathrm{~mm}$, y en algunas secciones $>0,6 \mathrm{~mm}$ (fenocristales), con macla tipo albita-carlsbad.

El clinopiroxeno representa entre el 30 y $50 \%$ de la roca, en cristales subhedrales a anhedrales, fracturados, incoloros, con bordes pardos por alteración; tamaño entre $0,1-0,5 \mathrm{~mm}$ hasta $1,5 \mathrm{~mm}$ en fenocristales, birrefringencia moderada de primer orden amarillo, y ángulo de extinción entre $40-50^{\circ}$, frecuentemente con extinción ondulatoria. El anfíbol es producto de la uralitización del piroxeno, caracterizado por hábito fibroso y masivo.

\section{GEOQUIMICA}

El análisis geoquímico de rocas diabásicas del Complejo Quebradagrande se compara con las Diabasas de San José de Urama y en algunos gráficos se presentan los campos de rocas de arco de la Formación Barroso y el Complejo Quebradagrande, datos que se extraen de los trabajos de Nivia et al., (2006); Rodríguez y Arango (2013a) y Rodríguez y Zapata (2013), con el objeto de mostrar las diferencias y similitudes entre rocas de corteza oceánica y entre rocas de arco asociadas.

En la TABLA 2 se muestran los resultados de óxidos mayores obtenidos en rocas diabásicas del Complejo Quebradagrande, con valores de $\mathrm{SiO}_{2}$ entre $44,95 \%$ y $50,80 \%$, concentraciones altas de $\mathrm{MgO}$ entre 5,42 y $8,9 \%$, valores de $\mathrm{Fe}_{2} \mathrm{O}_{3}$ entre 7,98 y $14,83 \%$, contenidos de $\mathrm{CaO}$ altos entre $7,55 \%$ y $13,2 \%$, valores de $\mathrm{TiO}_{2}$ en la mayoría de las muestras entre 1 y $2 \%$, más altos que en las rocas de las Diabasas de San José de Urama que presenta valores $<1 \%$ y solamente dos muestras (VR386R y VR364R) presentan valores menores a $1 \%$ en $\mathrm{TiO}_{2}$; tienen baja relación del álcalis vs $\mathrm{SiO}_{2}(0,03$ a $0,10 \mathrm{wt} \%$ ) y los valores de $\mathrm{K}_{2} \mathrm{O}$ son bajos de $0,07 \%$ a $0,47 \%$. Las pérdidas por calcinación en la mayoría de las muestras es del orden de $2,3 \%$ a $3,4 \%$ que se debe a alteración moderada y la muestra 95004 presenta perdidas por calcinación del orden de $6,62 \%$ indicando alta alteración que es corroborada por el análisis petrográfico (TABLA 2).

TABLA 2. Resultados analíticos para óxidos mayores (\%wt) en diabasas del Complejo Quebradagrande.

\begin{tabular}{ccccccccccccccccc}
\hline Muestra & $\mathbf{9 5 6 9 0}$ & $\mathbf{5 7 5 7}$ & $\mathbf{7 4 8 4}$ & $\mathbf{7 4 8 5}$ & $\mathbf{8 0 7 2 9}$ & $\mathbf{8 0 9 2 3}$ & $\mathbf{8 0 9 2 4}$ & $\mathbf{9 5 0 0 4}$ & $\mathbf{9 5 0 1 9}$ & $\mathbf{9 5 0 5 5}$ & $\mathbf{9 5 0 6 0}$ & $\mathbf{5 9 6 7}$ & VR386R VR364R \\
\hline $\mathrm{SiO}_{2}$ & 48,16 & 50,64 & 49,35 & 50,8 & 46,62 & 48,75 & 47,92 & 48,72 & 48,91 & 46,69 & 44,95 & 48,97 & 48,75 & 49,59 \\
$\mathrm{TiO}_{2}$ & 1,01 & 1,34 & 1,77 & 1,81 & 1,57 & 1,89 & 1,7 & 1,8 & 1,43 & 1,34 & 1,04 & 1,43 & 0,84 & 0,99 \\
$\mathrm{Al}_{2} \mathrm{O}_{3}$ & 14 & 17,65 & 13,56 & 12,82 & 14,61 & 13,28 & 14,03 & 14,09 & 14,13 & 14,05 & 16,02 & 13,03 & 14,31 & 14,35 \\
$\mathrm{Fe}_{2} \mathrm{O}_{3}$ & 10,84 & 7,98 & 11,49 & 11,71 & 11,98 & 12,64 & 14,34 & 11,63 & 10,46 & 12,18 & 11,16 & 14,12 & 10,29 & 10,58 \\
$\mathrm{MgO}$ & 8,82 & 5,42 & 8,26 & 7,59 & 8,98 & 8,03 & 6,55 & 5,8 & 8,24 & 8,15 & 8,1 & 7,91 & 8,9 & 8,65 \\
$\mathrm{CaO}$ & 11,1 & 8,06 & 9,51 & 8,92 & 10,34 & 9,88 & 8,84 & 7,55 & 10,79 & 12,71 & 13,17 & 7,97 & 12,47 & 13,2 \\
$\mathrm{Na}_{2} \mathrm{O}$ & 2,1 & 4,41 & 1,86 & 3,46 & 2,23 & 2,51 & 2,91 & 2,73 & 2,85 & 1,32 & 1,57 & 2,96 & 1,74 & 1,51 \\
$\mathrm{~K}_{2} \mathrm{O}$ & 0,42 & 0,47 & 0,35 & 0,1 & 0,07 & 0,09 & 0,39 & 0,39 & 0,3 & 0,07 & 0,25 & 0,29 & 0,12 & 0,11 \\
$\mathrm{P}_{2} \mathrm{O}_{5}$ & 0,076 & 0,219 & 0,171 & 0,156 & 0,129 & 0,159 & 0,127 & 0,18 & 0,122 & 0,098 & 0,08 & 0,144 & 0,06 & 0,08 \\
$\mathrm{MnO}$ & 0,02 & 0,02 & 0,02 & 0,02 & 0,03 & 0,03 & 0,03 & 0,02 & 0,02 & 0,02 & 0,02 & 0,02 & 0,16 & 0,16 \\
$\mathrm{LOI}$ & 3,15 & 3,38 & 3,29 & 2,32 & 3,17 & 2,41 & 2,74 & 6,62 & 2,43 & 3,06 & 3,33 & 2,94 & 2,3 & 0,7 \\
\hline
\end{tabular}


En el diagrama TAS (Le Bas et al., 1986) las diabasas del Complejo Quebradagrande y la unidad Diabasas de San José de Urama se distribuyen en el campo de los basaltos y algunas muestras alcanzan el campo de las andesitas basálticas (95004, 7485 y 6757), en la serie toleítica de afinidad subalcalina (FIGURA 3A). Las rocas de arco de la Formación Barroso y el Complejo Quebradagrande (en verde) caen dentro del campo de las andesitas basálticas, y las tobas de la Formación Barroso se agrupan en el campo de las andesitas con valores más altos de álcalis.

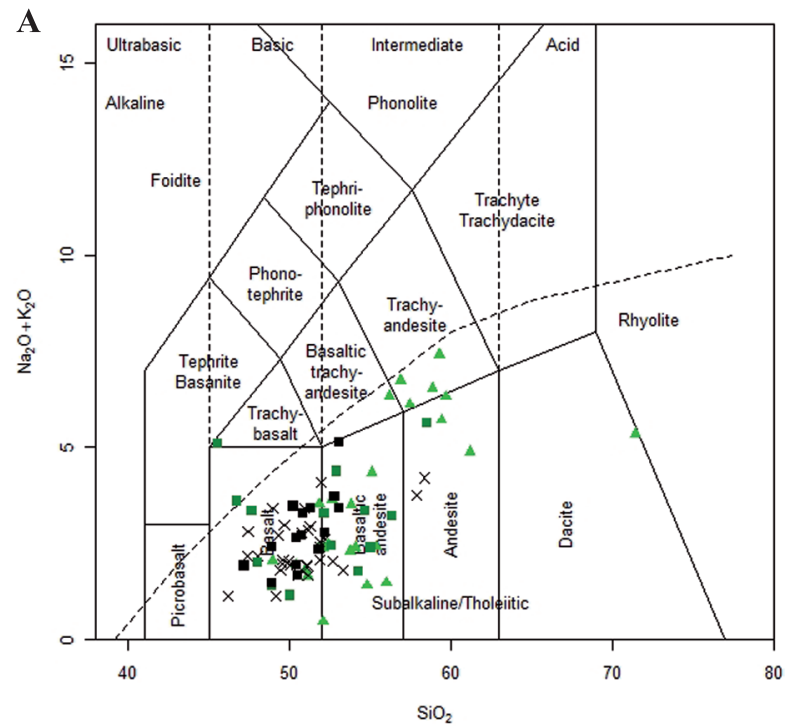

En el diagrama de clasificación de Hastie et al. (2007) las diabasas del Complejo Quebradagrande y las Diabasas de San José de Urama grafican en el campo de la serie toleítica con valores más altos de Co para las primeras y una mayor dispersión de datos que alcanzan el campo de la serie calcoalcalina. Las rocas de arco de la Formación Barroso ocupan los campos de los basaltos y las andesitas dentro de la serie toleítica, con una amplia dispersión, mientras las rocas de arco del Quebradagrande grafican en la serie calcoalcalina dentro de los campos de basaltos y andesitas con valores mayores de Th (FIGURA 3B).

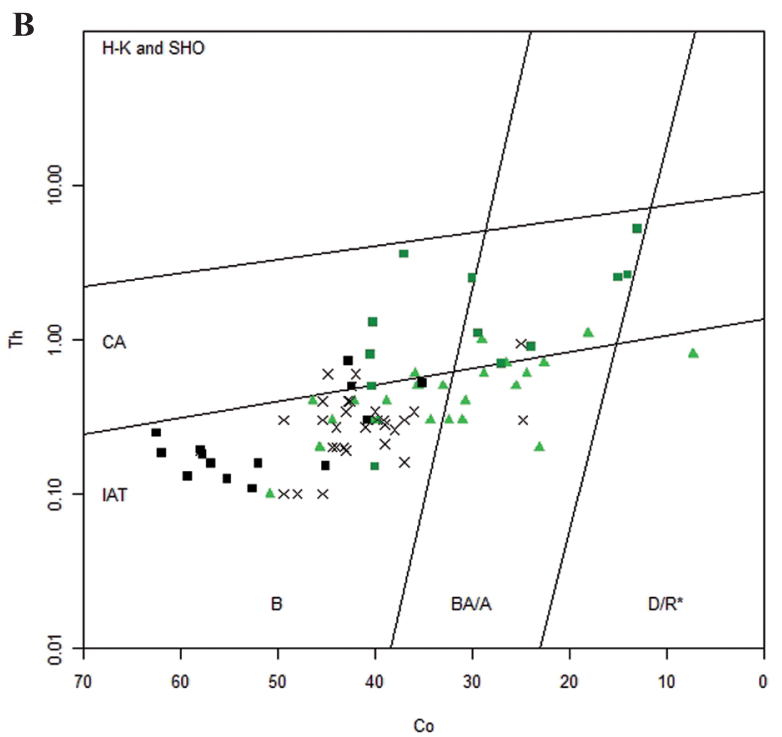

FIGURA 3. A. Diagrama TAS (Le Maitre et al., 1989). B. Diagrama de clasificación Th-Co (Hastie et al., 2007). Cuadros negros - diabasas del Complejo Quebradagrande, equis (x) Diabasas de San José de Urama, triángulos verdes - basaltos y tobas de arco de la Formación Barroso y cuadros verdes - basaltos y andesitas de arco del Complejo Quebradagrande.

En el diagrama de Jensen (1976) (FIGURA 4A), las diabasas del Complejo Quebradagrande y las Diabasas de San José de Urama se concentran en el campo de la serie de basaltos toleíticos ricos en $\mathrm{Mg}$, con algunas muestras dentro del campo de los basaltos toleíticos ricos en $\mathrm{Fe}$ (95024, 80024 y 95004); la muestra 5757 cae en el campo de los basaltos calcoalcalinos. Las rocas de arco se dispersan entre los basaltos komatíticos hasta las andesitas calcoalcalinas, concentrándose el mayor número de datos en los basaltos calcoalcalinos para la Formación Barroso y para las rocas de arco de Quebradagrande.

En el diagrama AFM de Irvine and Baragar (1971) -FIGURA 4B- las muestras de diabasas del Complejo Quebradagrande y las Diabasas de San José de Urama se distribuyen en el campo de las rocas toleíticas poco diferenciadas con bajo contenido de álcalis y únicamente la muestra 5757 cae en el campo calcoalcalino. Las rocas de arco del Complejo Quebradagrande se distribuyen en las rocas calcoalcalinas como en las toleíticas (datos tomados de Nivia et al., 2006; Villagómez et al., 2011; Rodríguez y Zapata, 2013).

La TABLA 3 muestra los resultados de elementos traza y tierras raras (REE) de diabasas del Complejo Quebradagrande. Los contenidos de $\mathrm{Nb}$ son similares a los valores encontrados en rocas de Diabasas de San José de Urama y solo dos rocas, VR-364R y 5757, presentan valores mayores de 4,40 ppm y 5,30 ppm, respectivamente.

Al comparar los valores de $\mathrm{K}_{2} \mathrm{O}, \mathrm{Sr}, \mathrm{Nb}$ y $\mathrm{Zr}$ de las diabasas (TABLA 4) con contenidos y valores en dorsales meso-oceánicas presentados por Wilson (1989), hay concordancia, en términos generales, entre 
los valores obtenidos en la mayoría de muestras y los generados en un ambiente tipo MORB. La mayoría de muestras presentan valores de $\mathrm{K}_{2} \mathrm{O}$ entre 0,1 y $0,3 \%$ y cinco muestras tienen valores entre $0,3 \%$ y $0,49 \%$ (9569, 5757, 7484, 80924, 95004); los valores de $\mathrm{Sr}$ para 10 muestras están entre $90 \mathrm{ppm}$ y $200 \mathrm{ppm}$, las muestras 5757, 90019, 95055, 95060 tienen $\mathrm{Sr}>200$ ppm y la muestra 80927 tiene contenido de Sr de 71,77 $\mathrm{ppm}$. Los contenidos de $\mathrm{Nb}, \mathrm{Zr}$ y las relaciones $\mathrm{Zr} / \mathrm{Y}$, $\mathrm{Nb} / \mathrm{Y},(\mathrm{La} / \mathrm{Yb}) \mathrm{N}$ y $(\mathrm{Gb} / \mathrm{Yb}) \mathrm{N}$ están dentro del rango de los MORB; los valores de la relación Ti/V son variables, pero la mayoría están dentro de los valores de 15 a 20 .
A

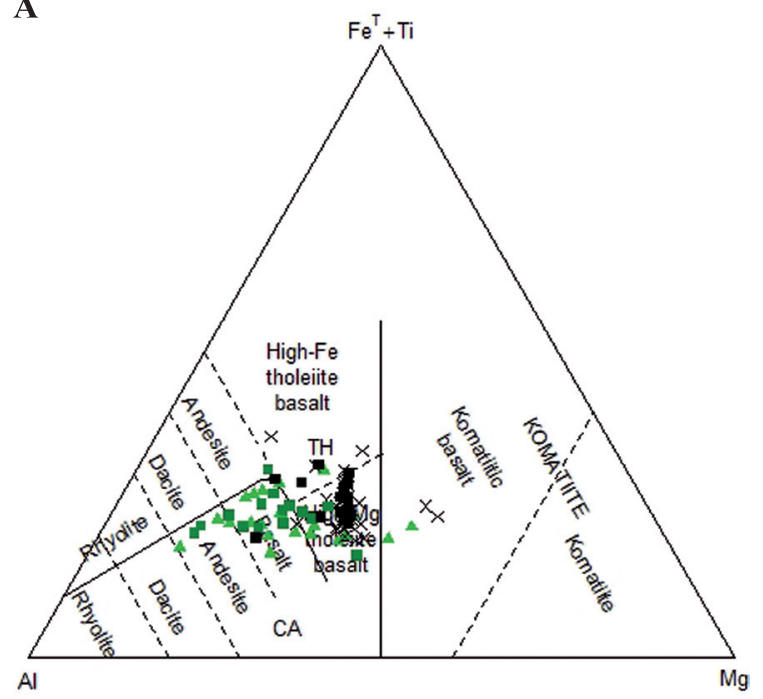

B

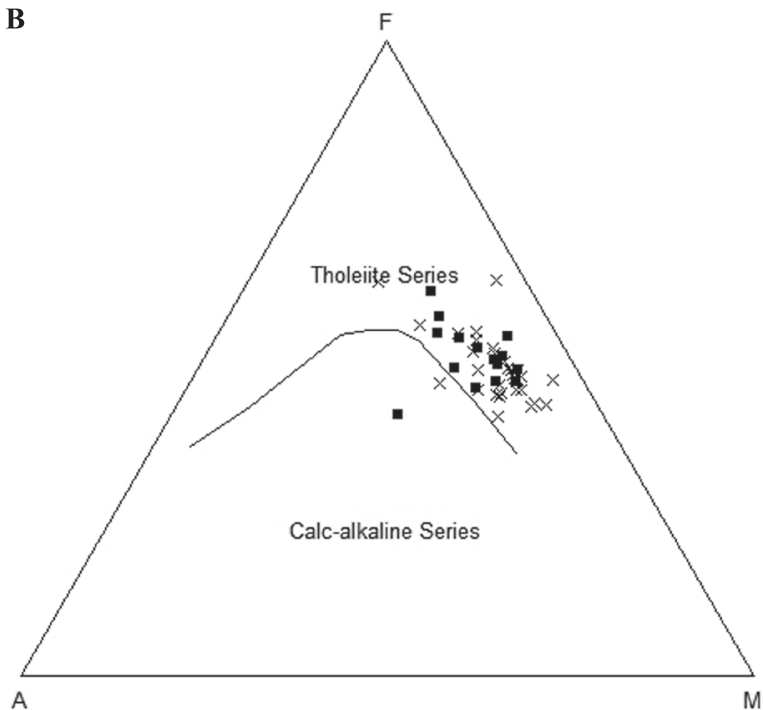

FIGURA 4. A. Diagrama de Jensen (1976). B. Diagrama AFM según Irvine and Baragar (1971). Cuadros negros - diabasas del Complejo Quebradagrande, equis (x) Diabasas de San José de Urama, triángulos verdes - basaltos y tobas de arco de la Formación Barroso y cuadros verdes - basaltos y andesitas de arco del Complejo Quebradagrande

Las mayores diferencias de las Diabasas de San José de Urama se presentan en las relaciones $\mathrm{Zr} / \mathrm{Nb}$ de 10 a 15 y $(\mathrm{La} / \mathrm{Sm}) \mathrm{N}$ con valores menores y mayores a 1 , resultado afín con ambientes de pluma, E-MORB o T-MORB, mientras en diabasas del Complejo Quebradagrande los valores de $\mathrm{Zr} / \mathrm{Nb}$ en su mayoría son mayores a 30 y la relación $(\mathrm{La} / \mathrm{Sm}) \mathrm{N}$ menor a 1 , sugiriendo afinidad con basaltos tipo N-MORB (basaltos de dorsales oceánicas normales o empobrecidos).

En los diagramas de las REE normalizados al condrito (valores de normalización de Nakamura, 1974), la mayoría de las muestras de diabasas del Complejo Quebradagrande tienen un comportamiento paralelo, con un patrón que sugiere afinidad con basaltos tipo N-MORB (FIGURA 5A), con empobrecimiento en tierras raras livianas $(\mathrm{La}, \mathrm{Ce})$ y tendencias planas horizontales hacia los elementos pesados. Las muestras VR-364R y VR-386R que se localizan espacialmente separadas del resto de muestras (ver FIGURAS 1 y
5) corresponden a las rocas más empobrecidas en tierras raras, con ligero enriquecimiento en tierras raras livianas $(\mathrm{La}, \mathrm{Ce})$ y tendencia plana horizontal hacia los elementos pesados, sugiriendo afinidad con corteza oceánica de tipo E-MORB. La muestra 5757 presenta el mayor enriquecimiento en tierras raras livianas (LREE) con relación a las tierras raras pesadas (HREE) y pendiente suave negativa, con un enriquecimiento mayor de diez veces el condrito, sugiriendo una probable fuente del arco toleítico para esta roca.

En el diagrama multielemental, las diabasas del Complejo Quebradagrande presentan mayor dispersión de los elementos más móviles (LILE), con anomalías positivas en $\mathrm{Cs}, \mathrm{Ba}, \mathrm{K}$ y $\mathrm{Sr}$ y tendencia plana para los elementos más inmóviles (HFSE) que en general tienen valores similares al N-MORB, con patrones planos entre $\mathrm{La} \mathrm{y} \mathrm{Lu}$ incluyendo el $\mathrm{Nb}$, y un patrón general de basaltos tipo MORB (FIGURA 5B) 
TABLA 3. Contenido de elementos traza y tierras raras en diabasas del Complejo Quebradagrande

\begin{tabular}{|c|c|c|c|c|c|c|c|c|c|c|c|c|c|c|}
\hline Muestras & 95690 & 5757 & 7484 & 7485 & 80729 & 80923 & 80924 & 95004 & 95019 & 95055 & 95060 & 5967 & VR386R & VR364R \\
\hline $\mathrm{Li}$ & 11,2 & 9,4 & 8,6 & 4,7 & 3,5 & 7,4 & 23,4 & 13,8 & 4,5 & 17,6 & 17,9 & 11,7 & & \\
\hline $\mathrm{Be}$ & 0,3 & 1 & 0,6 & 0,5 & 0,4 & 0,5 & 0,5 & 0,7 & 0,4 & 0,3 & 0,2 & 0,5 & 0 & 0 \\
\hline $\mathrm{Sc}$ & 54,3 & 31,7 & 48,5 & 45,8 & 53,6 & 50,9 & 57,5 & 46,7 & 44,9 & 54,1 & 53,6 & 43,6 & 42 & 43 \\
\hline $\mathrm{Co}$ & 57,7 & 35,1 & 59,3 & 52,6 & 59,3 & 55,2 & 62,5 & 52 & 56,9 & 58 & 62 & 45,1 & 40,8 & 42,4 \\
\hline $\mathrm{Ni}$ & 145,6 & 67,5 & 81 & 82,8 & 88,6 & 78 & 60,9 & 67,3 & 85,4 & 93,4 & 134,2 & 66 & 47 & 44,5 \\
\hline $\mathrm{Cu}$ & 129,3 & 48,8 & 169,7 & 32,4 & 98,5 & 78,4 & 157,7 & 71,4 & 87,4 & 135,7 & 136,8 & 60,9 & 37,2 & 132,6 \\
\hline $\mathrm{Zn}$ & 87,9 & 82,5 & 124,8 & 62,5 & 113,7 & 114,5 & 111,1 & 127,7 & 79 & 94,4 & 80 & 120,3 & 31 & 27 \\
\hline $\mathrm{Ga}$ & 15,2 & 18,5 & 19,2 & 14,2 & 19 & 19,1 & 18 & 18,9 & 18,2 & 18,8 & 16,5 & 17,6 & 13 & 14,3 \\
\hline As & 0,6 & 1,4 & 0,9 & 1 & 0,8 & 1,8 & 1,2 & 1 & 1,1 & 1,2 & 0,8 & 1,3 & 0,6 & 0 \\
\hline $\mathrm{Rb}$ & 8,4 & 9,1 & 3,3 & $<0,4$ & $<0,4$ & $<0,4$ & 11,1 & 9,4 & 1,4 & 0,6 & 5,4 & 5,7 & 2,3 & 1,3 \\
\hline $\mathrm{Sr}$ & 178,1 & 352,7 & 196,8 & 112,7 & 143 & 71,8 & 140 & 195 & 476,2 & 339,7 & 255,1 & 150,3 & 107,2 & 111,5 \\
\hline $\mathrm{Y}$ & 21,1 & 26,1 & 37 & 41,1 & 32,2 & 38,7 & 38,8 & 36,2 & 30 & 29,8 & 24,7 & 33,7 & 16 & 18,7 \\
\hline $\mathrm{Cd}$ & 0,2 & 0,2 & 0,2 & $<0,08$ & 0,1 & 0,3 & 0,3 & 0,3 & 0,2 & 0,2 & 0,2 & 0,2 & 0 & 0 \\
\hline In & 0,1 & 0,1 & 0,1 & 0,1 & 0,1 & 0,1 & 0,1 & 0,1 & 0,1 & 0,1 & 0,1 & 0,1 & & \\
\hline Cs & 0,5 & 0,4 & 0,1 & 0,1 & 0,1 & 0,1 & 1,1 & 0,2 & 0,1 & 0,1 & 0,3 & 0,4 & 0 & 0,6 \\
\hline $\mathrm{Ba}$ & 96,9 & 544,3 & 213,7 & 23,3 & 13,5 & 28,3 & 737,7 & 279,8 & 51,2 & 40 & 117,7 & 49,7 & 32,7 & 26,8 \\
\hline $\mathrm{La}$ & 2,4 & 9,9 & 4,9 & 4,5 & 3,2 & 4,8 & 3,7 & 4,2 & 3,2 & 3,2 & 2,2 & 4,2 & 2,7 & 4 \\
\hline $\mathrm{Ce}$ & 9,1 & 24,9 & 15,2 & 16,1 & 12,7 & 16,1 & 12,3 & 14,8 & 12,2 & 11 & 8,5 & 13 & 7,2 & 9,5 \\
\hline $\operatorname{Pr}$ & 1,4 & 3,8 & 2,6 & 2,7 & 2,2 & 2,8 & 2,2 & 2,5 & 2 & 1,8 & 1,4 & 2,2 & 1,1 & 1,4 \\
\hline $\mathrm{Nd}$ & 7,2 & 17,2 & 13,9 & 14,6 & 12 & 15,4 & 12,8 & 13,9 & 10,8 & 10,3 & 7,5 & 11,8 & 5,7 & 6,9 \\
\hline $\mathrm{Sm}$ & 2,6 & 4,7 & 4,9 & 5,2 & 4,4 & 5,3 & 4,7 & 4,9 & 3,9 & 3,7 & 2,8 & 4,2 & 1,6 & 1,9 \\
\hline $\mathrm{Eu}$ & 1 & 1,9 & 1,8 & 1,5 & 1,5 & 1,7 & 1,8 & 1,8 & 1,4 & 1,4 & 1,1 & 1,5 & 0,7 & 0,8 \\
\hline $\mathrm{Gd}$ & 3 & 4,8 & 5,5 & 5,6 & 4,8 & 5,8 & 5,5 & 5,4 & 4,2 & 4,2 & 3,2 & 4,7 & 2,2 & 2,7 \\
\hline $\mathrm{Tb}$ & 0,6 & 0,9 & 1,1 & 1,2 & 1 & 1,2 & 1,2 & 1,1 & 0,9 & 0,9 & 0,7 & 1 & 0,4 & 0,5 \\
\hline Dy & 4,3 & 5,5 & 7,4 & 7,8 & 6,7 & 7,9 & 7,9 & 7,5 & 5,8 & 5,9 & 4,8 & 6,4 & 2,6 & 3,1 \\
\hline Но & 0,9 & 1,1 & 1,6 & 1,6 & 1,4 & 1,7 & 1,7 & 1,6 & 1,2 & 1,2 & 1 & 1,4 & 0,6 & 0,7 \\
\hline $\mathrm{Er}$ & 2,7 & 3,1 & 4,5 & 4,7 & 4 & 4,8 & 4,9 & 4,3 & 3,6 & 3,6 & 3 & 3,9 & 1,7 & 2,1 \\
\hline $\mathrm{Tm}$ & 0,4 & 0,4 & 0,6 & 0,7 & 0,5 & 0,6 & 0,7 & 0,6 & 0,5 & 0,5 & 0,4 & 0,5 & 0,3 & 0,3 \\
\hline $\mathrm{Yb}$ & 2,3 & 2,4 & 3,7 & 4 & 3,3 & 3,7 & 4,2 & 3,2 & 3,1 & 3 & 2,6 & 3,1 & 1,5 & 1,8 \\
\hline $\mathrm{Lu}$ & 0,4 & 0,3 & 0,5 & 0,6 & 0,5 & 0,5 & 0,6 & 0,4 & 0,4 & 0,4 & 0,4 & 0,4 & 0,3 & 0,3 \\
\hline $\mathrm{Tl}$ & 0 & 0,2 & 0 & $<0,01$ & $<0,01$ & $<0,01$ & 0,1 & 0 & $<0,01$ & $<0,01$ & 0 & $<0,01$ & & \\
\hline $\mathrm{Pb}$ & $<2,0$ & $<2,0$ & $<2,0$ & $<2,0$ & $<2,0$ & 2,4 & $<2,0$ & $<2,0$ & $<2,0$ & $<2,0$ & $<2,0$ & $<2,0$ & 0 & 0,1 \\
\hline $\mathrm{Bi}$ & $<0,05$ & $<0,05$ & 0,1 & $<0,05$ & $<0,05$ & $<0,05$ & $<0,05$ & $<0,05$ & $<0,05$ & $<0,05$ & $<0,05$ & $<0,05$ & & \\
\hline Th & 0,2 & 0,5 & 0,1 & 0,1 & $<0,10$ & 0,1 & 0,2 & 0,2 & 0,2 & 0,2 & 0,2 & 0,2 & 0,3 & 0,5 \\
\hline $\mathrm{U}$ & 0,1 & 0,2 & 0,1 & 0 & 0,1 & 0,1 & 0,1 & 0,2 & 0 & 0,1 & 0,1 & 0,1 & 0,1 & 0 \\
\hline $\mathrm{V}$ & 275,6 & 174,9 & 334,2 & 309,7 & 284,9 & 353 & 364,1 & 328,2 & 264,9 & 305,9 & 270,7 & 266,7 & 263 & 299 \\
\hline $\mathrm{Cr}$ & 311,8 & 113,7 & 117,4 & 149,8 & 237,6 & 201,4 & 100,6 & 144,1 & 264,3 & 270,8 & 277,9 & 149,1 & & \\
\hline $\mathrm{Zr}$ & 50,1 & 141,7 & 102,7 & 108,5 & 86,9 & 112 & 89,2 & 104,7 & 84,4 & 75,5 & 55,6 & 80,4 & 38 & 46,4 \\
\hline $\mathrm{Nb}$ & 2,4 & 5,3 & 3,2 & 2,6 & 2,5 & 1,9 & 2,8 & 2,8 & 1,9 & 2 & 1,9 & 1,9 & 3,3 & 4,4 \\
\hline W & 8,3 & $<8$ & $<8$ & $<8$ & 9 & 8,3 & $<8$ & $<8$ & 11,4 & 11,5 & $<8$ & 8,8 & 0,1 & 0,1 \\
\hline $\mathrm{Tm}$ & 0,4 & 0,4 & 0,6 & 0,7 & 0,5 & 0,6 & 0,7 & 0,6 & 0,5 & 0,5 & 0,4 & 0,5 & 0,3 & 0,3 \\
\hline $\mathrm{Yb}$ & 2,3 & 2,4 & 3,7 & 4 & 3,3 & 3,7 & 4,2 & 3,2 & 3,1 & 3 & 2,6 & 3,1 & 1,5 & 1,8 \\
\hline $\mathrm{Lu}$ & 0,4 & 0,3 & 0,5 & 0,6 & 0,5 & 0,5 & 0,6 & 0,4 & 0,4 & 0,4 & 0,4 & 0,4 & 0,3 & 0,3 \\
\hline $\mathrm{Tl}$ & 0 & 0,2 & 0 & $<0,01$ & $<0,01$ & $<0,01$ & 0,1 & 0 & $<0,01$ & $<0,01$ & 0 & $<0,01$ & & \\
\hline $\mathrm{Pb}$ & $<2,0$ & $<2,0$ & $<2,0$ & $<2,0$ & $<2,0$ & 2,4 & $<2,0$ & $<2,0$ & $<2,0$ & $<2,0$ & $<2,0$ & $<2,0$ & 0 & 0,1 \\
\hline Th & 0,2 & 0,5 & 0,1 & 0,1 & $<0,10$ & 0,1 & 0,2 & 0,2 & 0,2 & 0,2 & 0,2 & 0,2 & 0,3 & 0,5 \\
\hline $\mathrm{U}$ & 0,1 & 0,2 & 0,1 & 0 & 0,1 & 0,1 & 0,1 & 0,2 & 0 & 0,1 & 0,1 & 0,1 & 0,1 & 0 \\
\hline $\mathrm{V}$ & 275,6 & 174,9 & 334,2 & 309,7 & 284,9 & 353 & 364,1 & 328,2 & 264,9 & 305,9 & 270,7 & 266,7 & 263 & 299 \\
\hline $\mathrm{Cr}$ & 311,8 & 113,7 & 117,4 & 149,8 & 237,6 & 201,4 & 100,6 & 144,1 & 264,3 & 270,8 & 277,9 & 149,1 & & \\
\hline $\mathrm{Zr}$ & 50,1 & 141,7 & 102,7 & 108,5 & 86,9 & 112 & 89,2 & 104,7 & 84,4 & 75,5 & 55,6 & 80,4 & 38 & 46,4 \\
\hline $\mathrm{Nb}$ & 2,4 & 5,3 & 3,2 & 2,6 & 2,5 & 1,9 & 2,8 & 2,8 & 1,9 & 2 & 1,9 & 1,9 & 3,3 & 4,4 \\
\hline W & 8,3 & $<8$ & $<8$ & $<8$ & 9 & 8,3 & $<8$ & $<8$ & 11,4 & 11,5 & $<8$ & 8,8 & 0,1 & 0,1 \\
\hline
\end{tabular}


TABLA 4. Comparación de la abundancia de algunos elementos y relaciones de elementos traza para rocas formadas en ambiente tipo MORB, plateau (Wilson, 1989) y valores obtenidos en diabasas del Complejo Quebradagrande y Diabasas de San José de Urama.

\begin{tabular}{ccccc}
\hline Elemento & MORB & Plateau & Diabasas Quebradagrande & $\begin{array}{c}\text { Diabasas San José de } \\
\text { Urama }\end{array}$ \\
\hline $\mathrm{K}_{2} \mathrm{O}$ & $<0,1-0,3$ & & 0,07 a 0,49 & 0,04 a 0,50 \\
$\mathrm{Sr}$ & $90-200$ & & 71,7 a 476 & 50,6 a 250,6 \\
$\mathrm{Zr}$ & $15-150$ & & 37,7 a 141,7 & 15,1 a 151 \\
$\mathrm{Nb}$ & 1 a 15 & 1 a 9 & 1,9 a 5,3 & 0,6 a 9,7 \\
$\mathrm{Zr} / \mathrm{Y}$ & $2 \mathrm{a} 4$ & 4 a 5 & 2,07 a 2,8 & 1,37 a 3,5 \\
$\mathrm{Nb} / \mathrm{Y}$ & $0,05-0,2$ & $0,23-0,95$ & 0,06 a 0,24 & 0,05 a 0,24 \\
$\mathrm{Ti} / \mathrm{V}$ & $15-20$ & $27-33$ & 14,51 a 30,4 & 6,8 a 20,9 \\
$(\mathrm{La} / \mathrm{Yb})^{\mathrm{N}}$ & $0,3-1,3$ & $1,2-4,5$ & 0,59 a 1,21 & 0,2 a 1,7 \\
$(\mathrm{Gd} / \mathrm{Yb})^{\mathrm{N}}$ & $0,9-1,4$ & $1,1-1,9$ & 1,04 a 1,6 & 0,8 a 1,4 \\
\hline
\end{tabular}
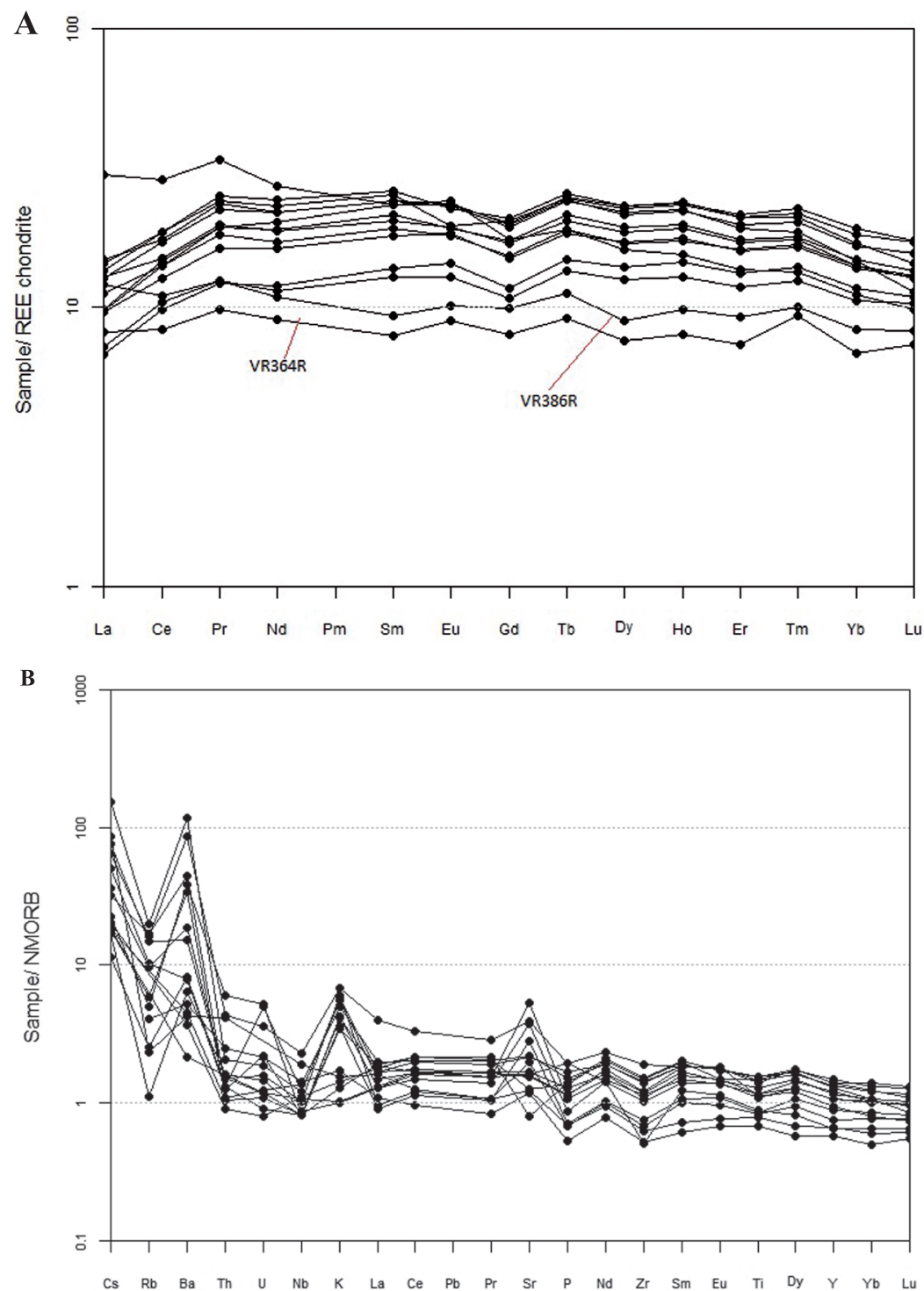

FIGURA 5. A. Diagramas de tierras raras (REE) normalizado con respecto al condrito (McDonough and Sun, 1995). B. Diagramas multielementales normalizados respecto al N-MORB (Sun and McDonough, 1989). 
En la FIGURA 6 y la TABLA 5 se hace la comparación de tierras raras de dos muestras de diabasas con contenidos similares de $\mathrm{MgO}(9,33 \%$ y $9,30 \%)$, una muestra de la Unidad Diabasas de San José de Urama (muestra IGM-706399) y una muestra de diabasas del Complejo Quebradagrande (muestra IGM-80729). La muestra IGM-80729 presenta un patrón similar a los N-MORB con empobrecimiento en las tierras raras livianas y relación $(\mathrm{La} / \mathrm{Yb}) \mathrm{N}$ de 0,66 , mientras la muestra IGM-706399 presenta un patrón plano similar a los T-MORB con relación $(\mathrm{La} / \mathrm{Yb}) \mathrm{N}$ de 0,87 , sugiriendo que corresponden a corteza oceánica de fuentes diferentes, siendo más empobrecida la diabasa del Complejo Quebradagrande.
La FIGURA 6B compara el patrón de tierras raras de dos muestras de diabasas del Complejo Quebradagrande (VR364R y IGM.7484) y una muestra de Diabasas de San José de Urama (JD-195R), con contenidos similares de $\mathrm{MgO}$ (8,72\%; 8,57\% y 8,72\%, respectivamente). Las muestras VR364R y JD-195R presenta un patrón paralelo y casi idéntico, con relación $(\mathrm{La} / \mathrm{Yb}) \mathrm{N}$ de 1,46 y 1,44 , respectivamente y patrón similar a T-MORB, por su parte la muestra IGM-7484 presenta un patrón similar a los N-MORB, con empobrecimiento en las tierras raras livianas y relación $(\mathrm{La} / \mathrm{Yb}) \mathrm{N}$ de 0,89 . Estos gráficos sugirieren que existen bloques que corresponden a corteza oceánica más empobrecidos y algunos bloques (muestras VR364R y VR386R9) podrían corresponder a la misma corteza de las Diabasas de San José de Urama.

TABLA 5. Valores de tierras raras normalizados de muestras de diabasa con valores de MgO similares. Muestras de Diabasas de San José de Urama (706399 y JD-195R) y muestras de diabasa del Complejo Quebradagrande (80729, VR-364R y 7484).

\begin{tabular}{|c|c|c|c|c|c|c|c|c|c|c|c|c|c|c|c|c|}
\hline & Elemento & La & $\mathrm{Ce}$ & $\operatorname{Pr}$ & Nd & Sm & Eu & Gd & Tb & Dy & Ho & Er & $\mathbf{T m}$ & $\mathbf{Y b}$ & Lu & $\mathbf{L a} / \mathbf{Y b}$ \\
\hline & Factor & 0,33 & 0,87 & 0,11 & 0,63 & 0,2 & 0,08 & 0,28 & 0,05 & 0,34 & 0,07 & 0,23 & 0,03 & 0,22 & 0,03 & \\
\hline M & 706399 & 7,17 & 6,92 & 7,86 & 7,57 & 7,64 & 7,58 & 8,62 & 9,79 & 8,43 & 8,71 & 8,36 & 9,33 & 8,23 & 8,29 & 0,87 \\
\hline $\begin{array}{l}\mathbf{u} \\
\mathbf{e}\end{array}$ & 80729 & 9,86 & 14,65 & 19,44 & 19,04 & 21,57 & 19,3 & 17,33 & 21,55 & 19,48 & 19,91 & 17,59 & 17,85 & 14,87 & 13,61 & 0,66 \\
\hline $\mathbf{s}$ & VR364R & 12,16 & 10,98 & 12,5 & 10,95 & 9,36 & 10,13 & 9,93 & 11,28 & 9,01 & 9,86 & 9,2 & 10 & 8,32 & 8,26 & 1,46 \\
\hline $\begin{array}{l}t \\
r\end{array}$ & 7484 & 14,9 & 17,55 & 23,63 & 22,01 & 24,21 & 23,55 & 19,82 & 24,3 & 21,51 & 22,22 & 19,87 & 20,17 & 16,72 & 15,62 & 0,89 \\
\hline a & JD-195R & 10,94 & 10,29 & 12,05 & 10,63 & 9,85 & 10,13 & 9,78 & 11,28 & 9,24 & 9,57 & 9,33 & 9,67 & 7,59 & 7,67 & 1,44 \\
\hline
\end{tabular}
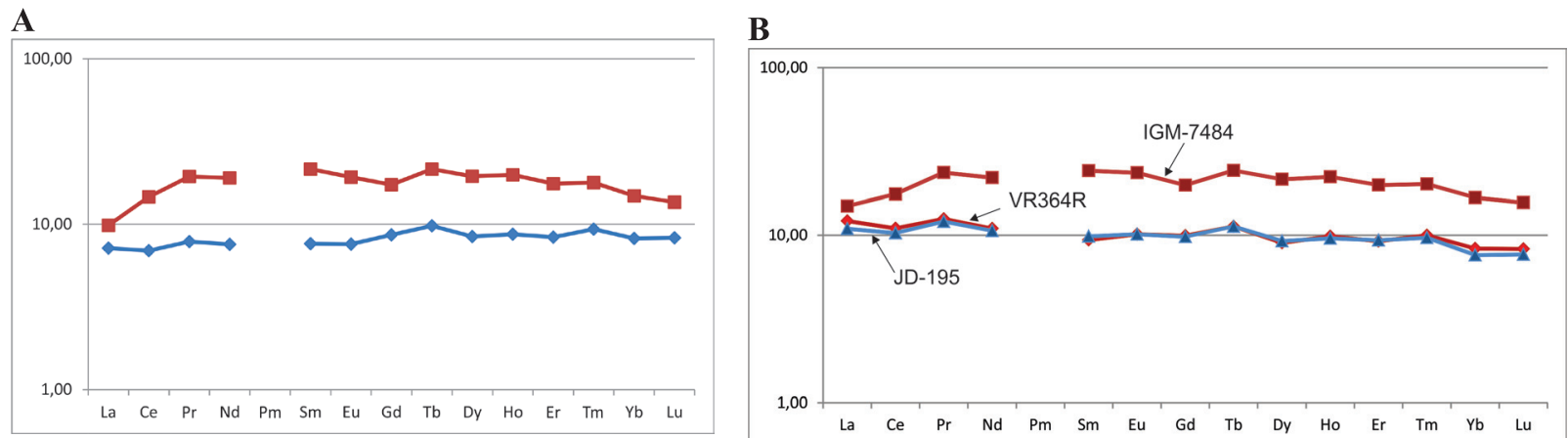

FIGURA 6. Patrón de tierras raras para muestras de diabasas del Complejo Quebradagrande y Diabasas de San José de Urama. A. En anaranjado muestra IGM-80729- Complejo Quebradagrande y en azul muestra IGM-706399- Diabasas de San José de Urama. B. En anaranjado muestra IGM-7484 y en rojo muestra VR384R del Complejo Quebradagrande; en azul muestra JD195R de la unidad Diabasas de San José de Urama.

El gráfico $\mathrm{Th} / \mathrm{Yb}-\mathrm{Nb} / \mathrm{Yb}$ (Pearce, 2008) permite definir el carácter oceánico de diferentes tipos de basaltos, los cuales grafican dentro del campo diagonal MORB-OIB de la FIGURA 7, con el eje principal de dispersión a lo largo de este campo. Los basaltos formados en márgenes continentales y en las zonas de subducción son comúnmente localizados dentro del gráfico por encima del campo MORB-OIB y prácticamente todos los magmas de arco grafican por encima del campo MORB-OIB (Pearce and Peate, 1995), aunque algunas lavas de cuencas de Back-Arc pueden aparecer por encima de este campo (Pearce, 2008).

La FIGURA 7 trae las áreas resultantes de los datos graficados por Pearce (2008) para rocas de corteza oceánica de la Cordillera Occidental (Plateau Colombia- 
línea punteada roja) y los basaltos de Gorgona (línea punteada negra); se adicionan los datos de diabasas del Complejo Quebradagrande y los datos de Diabasas de San José de Urama, éstos últimos publicadas por
Rodríguez y Arango (2013a), además, los datos de rocas de arco del Complejo Quebradagrande y de la Formación Barroso publicadas por Rodríguez y Arango (2013a) y Rodríguez y Zapata (2013).

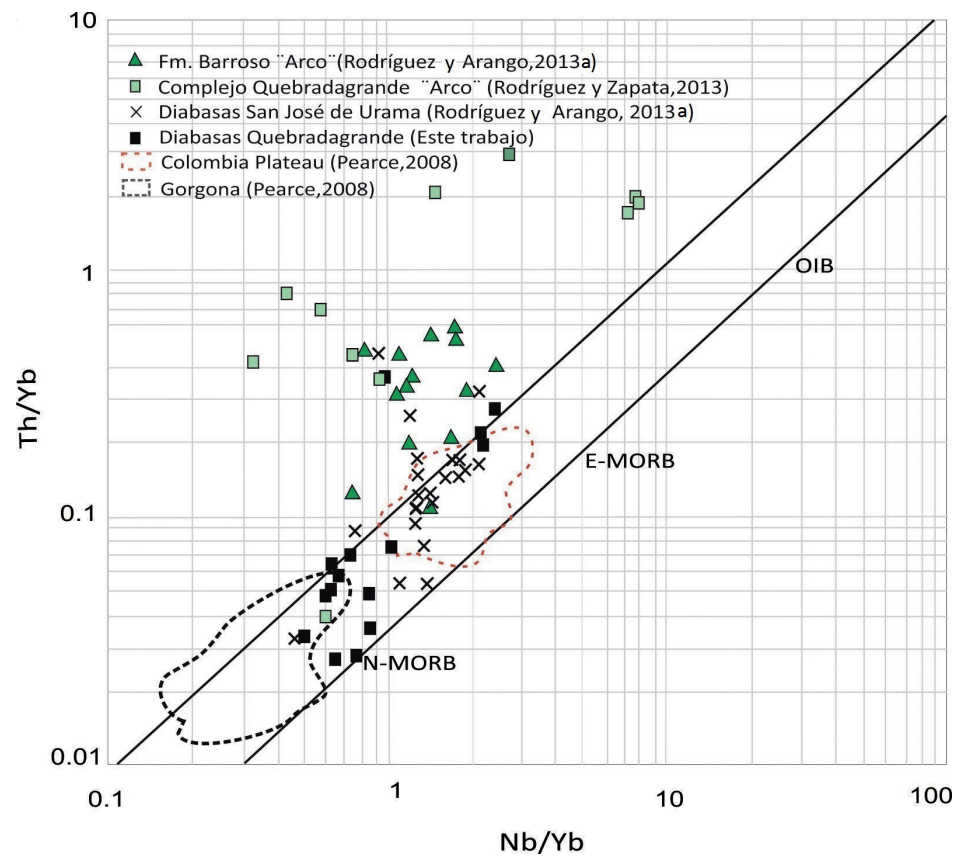

FIGURA 7. Diagrama de discriminación de ambiente para basaltos (Pearce, 1982). Manto empobrecido N-MORB, manto enriquecido E-MORB y basaltos de islas oceánicas (OIB). Cuadros negros - diabasas del Complejo Quebradagrande, equis (x) Diabasas de San José de Urama, triángulos verdes - basaltos de arco de la Formación Barroso y cuadros verdes - basaltos y andesitas de arco del Complejo Quebradagrande

Las diabasas del Complejo Quebradagrande grafican dentro del campo de los basaltos oceánicos (MORBOIB), con agrupación de los datos en dos poblaciones: una correspondiente a basaltos empobrecidos de tipo N-MORB y dos muestras (VR-364R y VR-384R) con enriquecimiento en $\mathrm{Th}$ y $\mathrm{Nb}$ que grafican dentro de los basaltos oceánicos de tipo E-MORB y en los bordes del campo del Plateau Colombia. Las muestras de Diabasas de San José de Urama corresponden a basaltos oceánicos que grafican la mayoría dentro del campo Plateau Colombia (campo definido a partir de los datos presentados por Pearce, 2008), intermedios entre N-MORB y E-MORB, sugiriendo que pueden ser parte del Plateau Colombia.

Las rocas de arco de la Formación Barroso y del Complejo Quebradagrande grafican por encima del campo de los basaltos oceánicos, confirmando el carácter de arco de estas rocas descrito por Nivia et al. (2006), Villagómez et al. (2011), Rodríguez y Arango (2013a), y Rodríguez y Zapata (2013), con diferencias entre ambos arcos, observándose una mayor dispersión de los datos de las rocas del Complejo Quebradagrande que podría deberse a más de un pulso magmático.
En el diagrama de Wood (1980), las diabasas del Complejo Quebradagrande presentan valores mayores de $\mathrm{Zr}$, la mayoría de las muestras grafican dentro del campo de los N-MORB y las muestras VR364R, VR386R presentan menores contenidos de $\mathrm{Zr}$ y grafican en el campo de los E-MORB, conjuntamente con las Diabasas de San José de Urama. Las Diabasas de San José de Urama se localizan en la interface de los campos N-MORB y E-MORB, siendo consideradas por Rodríguez y Arango (2013a) como rocas T-MORB (MORB transicional) (FIGURA 8).

La FIGURA 8 también presenta la distribución de rocas de arco del Complejo Quebradagrande (cuadros verdes) y de la Formación Barroso (triángulos verdes), con mayores valores en $\mathrm{Th}$ y menores en $\mathrm{Zr}$ y Nb para las rocas del Complejo Quebradagrade con relación a las rocas de la Formación Barroso, indicando que corresponden a rocas de arco más evolucionadas principalmente de arco calcoalcalino, y sugiriendo que son arcos diferentes. 


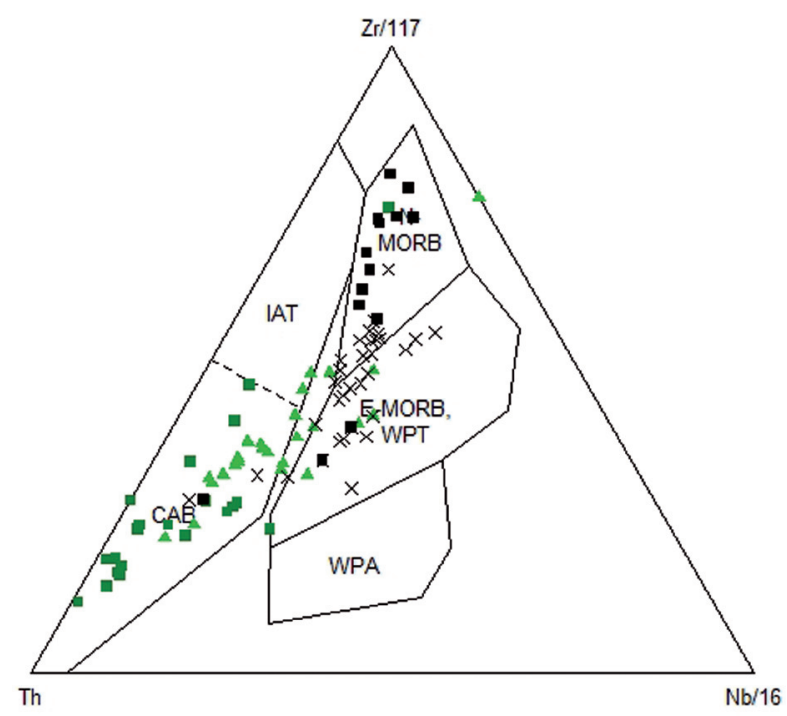

FIGURA 8. Diagramas $\mathrm{Zr} / 17-\mathrm{Th}-\mathrm{Nb} / 16$ (Wood, 1980) para muestras de diabasas del Complejo Quebradagrande (cuadros negros), Diabasas de San José de Urama (equis), rocas de arco del Complejo Quebradagrande (cuadros verdes) y rocas de arco de la Formación Barrosos (triángulos verdes).

\section{CONCLUSIONES}

El Complejo Quebradagrande está constituido por "bloques litológicos" de rocas sedimentarias marinas de edad Cretácico Temprano, rocas basálticas y andesíticas de arco (Nivia et al., 2006), que indican pulsos del Cretácico Temprano al Tardío (112 a 114 Ma- Pardo et al., 2011; Cochrane et al., 2014; Albiano y Campaniano - Jaramillo et al., 2015), junto a bloques de diabasas y basaltos de corteza oceánica de tipo N-MORB, y escasas muestras afines a basaltos oceánicos de tipo E-MORB.

Las características petrográficas de diabasas del Complejo Quebradagrande y de la unidad Diabasas de San José de Urama son similares y con los estudios que se adelantaron no se pueden indentificar diferencias petrográficas entre los bloques de diabasas que conforman ambas unidades. Por lo tanto, los bloques de corteza oceánica representados por diabasas y basaltos del Complejo Quebradagrande, presentan texturas intergranulares, ofíticas, subofíticas intersectales y variolíticas, similares a las rocas de corteza oceánica de la unidad Diabasas de San José de Urama.

Los valores de $\mathrm{TiO}_{2}$ en las diabasas del Complejo Quebradagrande varían entre 1 y $2 \%$ y solamente dos muestras (VR386R y VR364R) presentan valores menores a $1 \%$ en $\mathrm{TiO}_{2}$, siendo en general más altos que en las Diabasas de San José de Urama que tiene valores $<1 \%$ de $\mathrm{TiO}_{2}$.
Las diabasas del Complejo Quebradagrande fueron formadas por basaltos toleíticos altos en magnesio de la serie subalcalina, afines con corteza oceánica de tipo N-MORB y dos muestras (VR386R y VR364R) tienen afinidad con corteza oceánica de tipo E-MORB, sugiriendo la presencia de más de un tipo de corteza oceánica en los bloque litológicos que conforman el Complejo Quebradagrande. Al analizar la distribución espacial de las muestras y la afinidad geoquímica, parecen indicar la presencia de bloques de corteza oceánicas diferentes, espacialmente separados.

Las diabasas del Complejo Quebradagrande y unidades como las Diabasas de San José de Urama y el Grupo Diabásico (Plateau Colombia - Pearce, 2008) de la Cordillera Occidental, tienen una composición química de elementos trazas y tierras raras que sugiere diferentes cortezas oceánicas. La corteza oceánica en la Cordillera Occidental de Colombia (Grupo diabásico-Formación Volcánica) ha sido relacionada a un ambiente de pluma derivado de un plateau oceánico (Kerr et al., 1996, 1997) y afín a basaltos oceánicos de tipo T-MORB (Diabasas de San José de Urama - Rodríguez y Arango, 2013a), mientras las diabasas del Complejo Quebradagrande representan corteza oceánica de afinidad N-MORB asociada a una dorsal oceánica, exceptuando dos muestras (VR386R y VR364R) que presentan afinidad con E-MORB, las cuales grafican dentro del campo del Plateau Colombia y tienen un patrón similar a las Diabasas de San José de Urama. Estos resultados sugieren que las rocas de este complejo provienen posiblemente de al menos tres ambientes tectónicos: uno de convergencia con una zona de subducción que daría origen a las rocas de arco, otro de divergencia, con la generación de una dorsal que generaría las rocas de corteza oceánica representadas en la diabasas estudiadas en este trabajo, de afinidad N-MORB, probablemente asociadas a la extensión de una cuenca Back-arc y rocas formadas en ambiente de pluma derivado de un plateau oceánico que podría estar relacionados con las Diabasas de San José de Urama como lo muestra la geoquímica.

Las rocas de arco relacionadas al Complejo Quebradagrande muestran afinidad calcoalcalina y toleítica con una alta dispersión de los datos, tanto en óxidos mayores como en elementos traza y tierras raras, con un comportamiento diferente a las rocas de arco de la Formación Barroso, que están representadas por basaltos toleíticos subalcalinos menos evolucionados, indicando que muy probablemente ambos arcos (Arco Barroso y Arco Quebradagrande) fueron generados por eventos magmáticos diferentes, a través de cortezas oceánicas diferentes y no representan un mismo arco 
desmembrado como fue sugerido por Rodríguez y Zapata (2013), aunque la presencia de rocas de corteza oceánica de tipo E-MORB sugieren que existen bloques de rocas oceánica similares a las Diabasas de San José de Urama dentro del Quebradagrande y estos bloques podrían estar asociados a rocas de arco de la Formación Barroso.

Las diferencias químicas que presentan las rocas de corteza oceánica y las rocas de arco del Complejo Quebradagrande, con relación a las rocas de corteza oceánica y rocas de arco de las Diabasas de San José de Urama y la Formación Barroso, respectivamente, sugiere que ambas cortezas y ambos arcos se desarrollaron en general de manera independiente y la tectónica es la responsable de su posición actual.

\section{AGRADECIMIENTOS}

Se agradece al Servicio Geológico Colombiano por el apoyo prestado para desarrollar el trabajo de campo y laboratorio, así como el suministro de datos analíticos; y a los colaboradores y evaluadores del artículo por las valiosas observaciones y correcciones que permitieron mejorar el texto.

\section{REFERENCIAS}

Álvarez, E., y González, H. 1978. Geología y Geoquimica del Cuadrángulo I-7, Urrao. INGEOMINAS, Medellín, Informe $1761,347 \mathrm{p}$.

Álvarez, J. 1987. Tectonitas Dunitas de Medellín, Departamento de Antioquia, Colombia. Boletín Geológico INGEOMINAS, 28(3): 9-44.

Aspden, J. 1984. The Geology of the Western Cordillera and Pacific Coastal Plain in teh Departament of Valle del Cauca (Sheets 261, 278, 279, 280, and 299). INGEOMINAS, reporte No. 7, 61p.

Botero, G. 1963. Contribución al conocimiento de la geología de la Zona Central de Antioquia. Anales de la Facultad de Minas, pp. 57-101.

Cochrane, R., Spikings, R., Gerdes, A., Ulianov, A., Mora, A., Villagómez, D., Putlitz, B., and Chiaradia, M. 2014. Permo-Triassic anatexis, continental rifting and the disassembly of western Pangaea. Lithos, 190-191: 383-402.

GEOESTUDIOS-INGEOMINAS. 2005. Complementación geológica y geofísica de la parte occidental de las planchas 130 Santa Fé de Antioquia y 146 Medellín Occidental. Medellín.

González, H. 1976. Geología del Cuadrangulo J-8 (Sonsón). INGEOMINAS, Medellín, Informe 1704, 421p.

González, H. 2001. Mapa geológico del departamento de Antioquia, escala 1:400.000, memoria explicativa. INGEOMINAS, 240p.

Grosse, E. 1926. Estudio Geológico del Terciario Carbonífero de Antioquia. Dietrich Reimer, 361p. Berlín.

Hastie, A., Kerr, A., Pearce, J., and Mitchell, S. 2007. Classification of altered volcanic island arc rocks using immobile trace elements: development of theTh-Co discrimination diagram. Journal of Petrology, 48(12): 2341-2357.

Irvine, T., and Baragar, W. 1971. A guide to the chemical classification of the common volcanic rock. Canadian Journal of Earth Science, 8: 253-548.

Jaramillo, J.S., León-Vasco, S., Cardona, A., Valencia, V., y Vinasco, C. 2014. Complejo Quebradagrande: arco volcánico engrosado generado en un bloque continental con aporte de material Triásico. Resumen XI Semana Técnica de Geología e Ingeniería Geológica, UIS. Bucaramanga.

Jaramillo, J.S., León-Vasco, S., Cardona, A., Valencia, V., y Vinasco, C. 2015. Registro magmático del Complejo Quebradagrande: vestigios de la evolución tectónica entre el Albiano y el Campaniano en los Andes del Norte. Ponencia XV Congreso Colombiano de Geología. Bucaramanga.

Jensen, L. 1976. A new cation plot for classifying subalakalic volcanic rocks. Ontario Division Mines, Miscellaneous, 66p.

Kerr, A., Marriner, G., Arndt, N., Tarney, J., Nivia, A., Saunders, A., and Duncan, R. 1996. The petrogenesis of Gorgona komatiites, picrites and basalts: new field, petrographic and geochemical constraints. Lithos, 37(2): 245-260.

Kerr, A., Marriner, G., Tarney, J., Nivia, A., Saunders, A., Thirlwall, M., and Sinton, C. 1997. Cretaceous basaltic terranes in western Colombia: elemental chronological and $\mathrm{Sr}-\mathrm{Nd}$ constrainst on petrogenesis. Journal of Petrology, 38: 677-702. 
Le Bas, M., Le Maitre, R., Streckeisen, A., and Zannetin, B. 1986. A chemical classification of volcanic rocks based on the Total Alkali - Silica diagram. Journal of Petroleum Science and Engineering, 27: 745-750.

Le Maitre, R., Bateman, P., Dudek, A., Keller, J., Lameyre, J., Le Bas, M M.J., Sabine, P.A., Schmid, R., Sorensen, H., Streckeisen, A., Woolley, A.R., and Zanettin, B. 1989. A classification of igneous rocks and glossary or terms: recommendations of the International Union of Geological Sciences Subcommission on the systematic of igneous rocks. Blackwell Scientific Publications, Oxford, U.K, 193p.

Mejía, M., y Salazar, G. 1989 (publicado 2007). Memoria explicativa de la Geología de la Plancha 114 (Dabeiba) y parte W de la 115 (Toledo). Escala 1:100.000. INGEOMINAS, Medellín. 111p.

McDonough, W. F., and Sun, S. S. 1995. The composition of the Earth. Chemical Geology, 120(3): 223-253.

Moreno-Sánchez, Gómez-Cruz, A., y Toro, L. 2008. Proveniencia del material clástico del Complejo Quebradagrande y su relación con los complejos estructurales adyacentes. Boletín Ciencias de la Tierra, 22: 27-38.

Nakamura, N. 1974. Determination of REE, Ba, Fe, $\mathrm{Mg}, \mathrm{Na}$ and $\mathrm{K}$ in carbonaceous and ordinary chondrites. Geochimica et Cosmochimica Acta, 38(5): 757-775.

Nelson, H. 1957. Contribution to the geology of the Central and Western Cordillera of Colombia in the sector between Ibagué and Cali. Leidse Geologische Mededelingen. 22: 1-76.

Nivia, A., Marriner, G., y Kerr, A. 1996. El Complejo Quebradagrande una posible cuenca marginal intracratónica del Cretáceo Inferior en la Cordillera Central de los Andes. VII Congreso Colombiano de Geología, Memoria 3, pp. 108-123.

Nivia, A., Marriner, G., Kerr, A., and Tarney, J. 2006. The Quebradagrande Complex: A Lower Cretaceous ensialic marginal basin in the Central Cordillera of the Colombian Andes. Journal or South American Earth Sciences. 21: 423-436.

Pardo-Trujillo, A., Cardona, A., Silva, J. C., Borrero, C., y Tamayo, J. E. 2011. Geocronología U/Pb en circones detríticos del Complejo Quebradagrande: nuevos datos sobre la procedencia de los sedimentos cretáceos en la margen NW de Suramérica. XIV Congreso Latinoamericano de Geología y XIII Congreso Colombiano de Geología.
Pearce, J. 1982. Trace element characteristics of lavas from destructive plate boundaries. En Thorpe R.S. (ed.) Andesites, pp. 525-548.

Pearce, J. 2008. Geochemical fingerprinting of oceanic basalts with applications to ophiolite classification and the search for Archean oceanic crust. Lithos, 100: 14-48.

Pearce, J., and Peate, D. 1995. Tectonic implications of the composition of volcanic arc magmas. Earth and Planetary Science, 23: 251-85.

Rodríguez, G., y Arango, M. 2013a. Formación Barroso: arco volcánico toleítico y Diabasas de San José de Urama: un prisma acrecionario T-Morb en el segmento norte de la Cordillera Occidental de Colombia. Boletín Ciencias de la Tierra, 33: 17-38.

Rodriguez, G., y Arango, M. 2013b. Reinterpretación geoquímica y radiométrica de las matabasitas del Complejo Arquía. Boletín de Geología, 35(2): 65-81.

Rodríguez, G., y Zapata, G. 2013. Análisis comparativo entrelaFormaciónBarrosoyelComplejoQuebradagrande: un arco volcánico toleítico-calcoalcalino, segmentado por el sistema de fallas de Romeral en los Andes del Norte? Boletín Ciencias de la Tierra, 33: 39-58.

Rodríguez, G., Zapata, G., y Gómez, J. 2010. Geología de la parte oriental de la plancha 114 Dabeiba. INGEOMINAS, 172p.

Sun, S., and McDonough, W. 1989. Chemical and isotropic systematics of oceanic basalts:implications for mantle composition and processes. Magmatism in ocean basin, Geology Society Special Publication, No 42; London, pp. 313-345.

Villagómez, D., Spikings, R., Magna, T., Kammer, A., Winkler, W., and Beltrán, A. 2011. Geochronology, geochemistry and tectonic evolution of the Western and Central cordilleras of Colombia. Lithos, 125: 875-896.

Wilson, M. 1989. Igneous petrogenesis: a global tectonic approach. Unwin Hyman (editor), London, 466p.

Wood, D. 1980. The aplication of Th-Hf-Ta diagram to problems of tectonomagmatic classification and establishing the nature of cristal contamination of basaltic lavas of rhe British Tertiary volcanic province. Earth Planet Science Letters, 50: 11-30.

Trabajo recibido: septiembre 15 de 2015

Trabajo aceptado: marzo 18 de 2016

Manuscrito publicado en internet: abril 4 de 2016 